\title{
Factores personales que inciden en el bajo rendimiento académico de los estudiantes de geometría
}

\author{
Personal Factors that have an impact on the low academic \\ performance of Geometry student
}

Niorka Medina*; Johan Fereira ${ }^{* *}$ y Robinson Marzol ${ }^{* * *}$

\section{RESUMEN}

El objetivo de este artículo es analizar los factores personales percibidos en los estudiantes de geometría que intervienen en su bajo rendimiento académico. En cuanto a los referentes teóricos, los principales autores citados son Tejedor y GarcíaValcárcel (2007), Montes y Lerner (2011).Se enmarcó en el enfoque positivista, con una metodología cuantitativa, de tipo descriptivo, con un diseño no experimental y transversal de campo. La muestra fueron 200 estudiantes de la cátedra Geometría de la Facultad de Ingeniería de La Universidad del Zulia. Para la recolección de datos se aplicó un cuestionario dirigido a los estudiantes, constituido en la sección de Factores Personales por 8 ítems de selección simple y escala de tipo Likert. Para el tratamiento estadístico de los datos se utilizó distribución de frecuencias, así como las medias aritméticas ubicando los resultados en el baremo de la investigación. Los resultados indicaron que los factores personales intervienen medianamente en el bajo rendimiento de los estudiantes de Geometría; sin embargo se destacan la alta intervención que tienen situaciones personales significativas y el establecimiento de relaciones en pareja en este bajo rendimiento. Como conclusión se determinó la necesidad de implementar estrategias didácticas para optimizar el rendimiento académico de los estudiantes de geometría, presentándoles mejores incentivos que genere motivación y logre en los estudiantes un mayor compromiso para el éxito de

\footnotetext{
*Doctora en Ciencias de la Educación, Magister Scientiarum en Matemática Aplicada, Ingeniero en Computación, Profesora Titular a Dedicación Exclusiva de la cátedra de Cálculo II del Departamento de Matemática de la Facultad de Ingeniería de la Universidad del Zulia. Maracaibo-Venezuela. Correo electrónico: niorkamedina@ gmail.com

** Magister Scientiarum en MatemáticaAplicada, Licenciado en Educación Mención Matemática y Física, Profesor Asociado a Tiempo Completo de la cátedra de Geometría del Departamento de Matemática de la Facultad de Ingeniería de la Universidad del Zulia. Maracaibo-Venezuela. Correo electrónico: jfereira13@ hotmail.com

${ }^{* * * *}$ Magister Scientiarum en MatemáticaAplicada, Licenciado en Educación Mención Matemática y Física, Profesor Agregado a Tiempo Completo de la cátedra de Cálculo I del Departamento de Matemática de la Facultad de Ingeniería de la Universidad del Zulia. Maracaibo-Venezuela. Correo electrónico: rmarzol@ hotmail.com
} 
sus estudios. Asimismo, se sugirió realizar investigaciones sobre las causas del bajo rendimiento académico de estos estudiantes abordando todos los factores involucrados con la participación de los docentes, autoridades y del propio estudiante.

Palabras claves: Bajo Rendimiento Académico, Factores Personales, Estudiantes de Geometría, Desempeño Estudiantil.

\title{
RECIBIDO: Marzo 2017 ACEPTADO: Octubre 2017
}

\begin{abstract}
The objective of this article is to analyze the personal factors perceived by students of geometry that intervene in their low academic performance. As for the theoretical references, the main authors cited are Tejedor and García-Valcárcel (2007), Montes and Lerner (2011). It was framed in the positivist approach, with a quantitative methodology, of descriptive type, with a non-experimental and transversal field design. The shows were 200 students of the chair Geometry of the Faculty of Engineering of the University of Zulia. For the data collection, a questionnaire addressed to the students was applied, constituted in the section of Personal Factors by 8 items of simple selection and scale of Likert type. For the statistical treatment of the data, it was used frequency distribution, as well as the arithmetic means, placing the results in the scale of the research. The results indicated that the personal factors intervene moderately in the low performance of the students of Geometry; However are highlighted the high intervention that have significant personal situations and the establishment of relationships in couple in this underperformance. In conclusion, was determined the need to implement didactic strategies to optimize the academic performance of students of geometry, presenting them with better incentives that generate motivation and achieve in students a greater commitment to the success of their studies. Likewise, it is suggested to perform research on the causes of the low academic performance of these students, addressing all the factors involved with the participation of teachers, authorities and the student himself.
\end{abstract}

Keywords: Low academic performance, Personal Factors, Students of Geometry, Student Performance.

\section{Introducción}

El rendimiento académico de los estudiantes universitarios, según Guzmán (2012), constituye un factor imprescindible para valorar la eficacia de la educación superior; además es considerado el más claro indicador de la calidad educativa en la 
Factores personales que inciden en el bajo rendimiento académico de los estudiantes de geometría

enseñanza universitaria. Tomando en cuenta la limitación de los recursos gubernamentales para la educación superior pública, se hace necesario realizar investigaciones sobre el desempeño estudiantil, las cuales informaran sobre la realidad estudiantil, representando un insumo valioso en la toma de decisiones para las instituciones universitarias y, para el Estado, un indicador de inversión.

En consecuencia, las instituciones de educación superior a nivel mundial están sumergidas en un proceso de transformación, para dar respuesta a los diversos inconvenientes que enfrentan, entre los que se encuentra, según Boyer y Pérez (2011), el bajo rendimiento estudiantil. De acuerdo con Miranda, Montaño y Sáez (2012), representa un inconveniente para los responsables de la política educativa, debido al incremento del gasto público que ocasiona además de incidir negativamente en la evaluación de la calidad de la educación superior en un país. Para Martí (2012), esta situación podría suponer un obstáculo para el financiamiento de la implantación de mejoras educativas ante una situación económica de restricciones presupuestarias.

Así lo afirma Báez (2013), quien manifiesta que abandonar los estudios superiores y los bajos rendimientos tiene un tremendo costo económico y social. Según este autor se ve afectada la familia, pues tendrá que prolongar el pago de la educación de su hijo; se afecta el Estado, porque asigna becas o créditos mediante fondos gubernamentales; se afecta el estudiante, debido a que posterga su sueño profesional y su incorporación al campo de trabajo. Finalmente, es un costo social, ya que las personas con menos preparación resultan ser menos productivas y más propensas a romper las reglas por las que se rige la sociedad.

Hoy en día, la medición del rendimiento académico y su predicción mediante los factores que lo determinan, representan el tema central para muchas investigaciones en instituciones de educación superior.Para Tejedor y GarcíaValcárcel (2007), la mayoría de los estudios nacionales e internacionales sobre 
Niorka Medina; Johan Fereira y Robinson Marzol

Telos Vol. 20, No. 1 (2018). 4-28.

rendimiento estudiantil indican que los abandonos se producen en los primeros años de carrera, acentuándose en el primer año (en torno al 65\% de abandono).

La asignatura Geometría, de la Facultad de Ingeniería de LUZ, está ubicada en el primer semestre, y comprende el estudio de la geometría métrica y analítica del plano; además hace énfasis en el uso del razonamiento formal y sistemático para la resolución de problemas. Asimismo, la geometría es una parte importante de la cultura del hombre, es considerada como un mecanismo utilizado para encontrar soluciones a los problemas más comunes de quienes la han aplicado en su vida, pues, entre otros usos, facilita la medición de estructuras sólidas reales, tanto tridimensionales como superficies planas y es bastante útil para la realización de complejas operaciones matemáticas.

De acuerdo con Pitalúa (2012), su estudio demanda de orden, método, claridad, precisión y concatenación perfecta del saber, requiriéndose una precisión en los conocimientos al momento de resolver problemas, utilizar el lenguaje de manera rigurosa y concatenada, necesitándose de un dominio en la comprensión, abstracción y memoria, es decir en las operaciones geométricas fundamentales.

Ahora bien, según información suministrada por el Departamento de Matemática de la Facultad de Ingeniería de la Universidad del Zulia, el promedio porcentual de estudiantes aplazados y sin información de geometría en dicha facultad en los últimos tres años, se han ido incrementando superando el $50 \%$ de los estudiantes inscritos; así pues para el año 2011 se obtuvo un 50,86\% de aplazados y un 25,49\% sin información; para el año 2012 el porcentaje de aplazados alcanzó el $50,33 \%$ y el porcentaje de sin información el 21,57\%; para el 2013 el porcentaje de aplazados logró el $45,83 \%$ y el porcentaje de sin información el 34,75\%; mientras que para el primer periodo del 2014 el porcentaje de aplazados alcanzó el 55,67\% y el porcentaje de sin información el 27,65\%.En líneas generales, tales resultados respaldan la gran preocupación que manifiesta el Departamento de Matemática con respecto al rendimiento estudiantil en la asignatura de Geometría. 
Factores personales que inciden en el bajo rendimiento académico de los estudiantes de geometría

La Universidad del Zulia cuenta con un Reglamento de Evaluación y Rendimiento Estudiantil, estipulando en su artículo 2 que la finalidad de la evaluación del rendimiento estudiantil consiste en proporcionar evidencias válidas y confiables que permitan al profesor valorar el aprovechamiento del estudiante, determinar las causas de los resultados insatisfactorios y establecer las reorientaciones necesarias para que el alumno mejore sus logros académicos; y en su artículo 11 manifiesta que la escala de calificaciones en la que se evaluará tal rendimiento será de cero a veinte puntos y se requerirá una calificación mínima aprobatoria de diez puntos (Consejo Universitario de la Universidad del Zulia, 2000).

En la Facultad de Ingeniería existe el Sistema de Evaluación de los Aprendizajes, cuyo artículo 6 reza que las Cátedras programarán al menos tres evaluaciones ordinarias las cuales deberán realizarse para evaluar objetivos trazados por las asignaturas, donde cada evaluación ordinaria representará una calificación parcial, y en su artículo 9 se indica que la calificación definitiva será el promedio ponderado de las calificaciones parciales obtenidas de acuerdo al peso que ellas tengan asignadas por las Cátedras (Secretaria del Consejo de la Facultad de Ingeniería, 2001).

Todos estos reglamentos estan enmarcados en la Ley Orgánica de Educación, cuyo artículo 63 expresa que la evaluación como parte del proceso educativo “.... deberá apreciar y registrar de manera permanente, mediante procedimientos apropiados, el rendimiento del educando, tomando en cuenta los factores que integran su personalidad, valorará asimismo la actuación del educador y, en general, todos los elementos que constituyen dicho proceso"(Congreso de la República de Venezuela, 1999, p.19).

En pocas palabras, según Boyer y Pérez (2011, p.8), "todo proceso de evaluación curricular implica, una evaluación del rendimiento académico, pues ello permite vislumbrar los objetivos curriculares alcanzados". Para aprobar una asignatura 
Niorka Medina; Johan Fereira y Robinson Marzol

Telos Vol. 20, No. 1 (2018). 4-28.

se exige 10 puntos, disminuyendo a un 50\% la cantidad de objetivos que un estudiante debe alcanzar, considerándose únicamente la adquisición de conocimientos más no el desarrollo de sus capacidades intelectuales.

En función de la problemática planteada el presente artículo tiene como propósito analizar los factores personales percibidos por los estudiantes de geometría que intervienen en su bajo rendimiento académico. Para la elaboración de este artículo se consideraron los resultados de la investigación denominada "Causas del Bajo Rendimiento de los estudiantes de geometría en Ingeniería" cuyo propósito fue determinar las razones que inciden en el bajo rendimiento académico en la asignatura de geometría de los estudiantes de Ingeniería de la Universidad del Zulia, desde el punto de vista de los estudiantes. Considerando para su estudio los siguientes factores: personales, económicos-familiares, académicos e institucionales. Para finalmente, formular lineamientos que permitan mejorar el rendimiento académico de tales estudiantes en dicha asignatura facilitando así la calidad del aprendizaje.

Factores Personales que inciden en el bajo rendimiento académico

El rendimiento académico se define como "un indicador evaluador del avance de los conocimientos que va adquiriendo el estudiante en su proceso de aprendizaje reflejado en una cartilla a través de cantidades numéricas, mismo que sirve para ir corrigiendo las falencias detectadas" (Martínez y Paladinez, 2016, p.29). Para estas autoras, este indicador es de gran significancia pues describe el nivel de logro alcanzado por el estudiante.

García (2013, p. 46) comparte la idea anterior al considerar que el Rendimiento Académico es "el encargado de indicar la valoración de las capacidades y conocimientos logrados dentro del proceso de enseñanza-aprendizaje; es decir, lo que el estudiante obtenga como resultado al finalizar determinada materia así se estará midiendo su rendimiento académico".

Por otro lado, el bajo rendimiento académico significa que el estudiante no ha adquirido de forma adecuada y completa los conocimientos, además de que no posee 
Factores personales que inciden en el bajo rendimiento académico de los estudiantes de geometría

las herramientas y habilidades necesarias para la solución de problemas referente al material de estudio (García-Cruz; Guzmán y Martínez, 2007). De acuerdo con Barahona (2014, p.26), "las causas del bajo rendimiento académico deben buscarse más allá del estudiante mismo”. Para Martínez y Paladinez (2016), el bajo rendimiento académico es una representación de escasos niveles de participación en el proceso de aprendizaje, el cual se ve influenciado por distintos factores, reflejándose en una nota bien sea cualitativa o cuantitativa; así pues "es una característica negativa del rendimiento académico" (p.34).

En Latinoamérica, los estudiantes están rezagados en cuanto al rendimiento estudiantil, comparado con los países industrializados ya que nos encontramos con una clase social que no alcanza los conocimientos básicos para enfrentarse a las exigencias de la educación superior. Venezuela, desafortunadamente, no se escapa de esta realidad, pues a nivel de la educación superior tiene un grave problema de bajo rendimiento estudiantil, motivando así su análisis en diferentes casas de estudios universitarios. Tal análisis respaldará lo que Tapia y Tapia (2012) expresan sobre la necesidad de conocer las deficiencias en el tema del rendimiento estudiantil pues representaría el mejor recurso para corregirlas; ya que el estudiante podrá fortalecer la autorrealización, desarrollar la autonomía personal, sentirse satisfechos, felices y, así, no ser personas conflictivas.

De esta manera, Liprandi y otros (1993) citado por Hernández (2005), manifiestan que en la Educación Superior Venezolana, el cincuenta por ciento de los estudiantes universitarios fracasa y es en los cuatro primeros semestres de sus estudios cuando se incrementa la repitencia. Además, el problema del bajo rendimiento académico, deserción se agrava en mayor medida, en aquellas carreras universitarias, que exigen del pensamiento lógico abstracto.

En las instituciones universitarias se determinan criterios evaluativos propios, para obtener un promedio ponderado de las asignaturas que cursa el estudiante, 
Niorka Medina; Johan Fereira y Robinson Marzol

Telos Vol. 20, No. 1 (2018). 4-28.

tomando en cuenta elementos como la cantidad de asignaturas, el número de créditos y el valor obtenido en cada una de ellas; así pues las notas obtenidas, como lo indican Rodríguez, Fita, Torrado (2004) citados por Garbanzo (2007, p.46), representan "un indicador preciso y accesible para valorar el rendimiento académico", asumiendo que éstas muestran los alcances del estudiante en aspectos personales, académicos y sociales; además son resultado de factores tanto de tipo personal del estudiante, como didácticas del docente, contextuales e institucionales, los cuales finalmente influyen en el desempeño estudiantil.

Tomando en cuenta las principales investigaciones, en el área universitaria, en las que se ha contemplado la regularidad académica de los estudiantes o sus calificaciones, se puede concluir que las bajas calificaciones, los altos porcentajes al no presentar un examen y la alta tasa de repetición, no son un fenómeno reciente.

De hecho, en un estudio desarrollado en la Universidad de Salamanca por el grupo "Helmántica" se consideró la percepción y opinión de los estudiantes universitarios encuestados sobre las causas del bajo rendimiento académico, distinguiéndose entre las Causas personales: ausencia de aptitudes y capacidades, poco dominio de técnicas de estudio, escasa o falta de dedicación a sus estudios, durante el periodo de clases; así como la inasistencias a clases, desorganización del tiempo y planificación del estudio; ausencia de autoexigencia o de sentido de responsabilidad, insatisfacción con la carrera elegida, falta de proporción entre esfuerzos y resultados, problemas personales (García-Cruz, Guzmán y Martínez, 2007).

En ese mismo orden de ideas, Barahona (2014) indica que las calificaciones obtenidas por los estudiantes en la universidad son en gran medida influenciadas por las características individuales del individuo y que los factores relacionados con su desempeño académico pueden provenir de: determinantes personales y determinantes sociales. En el primero de ellos se incluye: la inteligencia, las aptitudes, la asistencia a clases, el género, la nota de acceso a la universidad. En el segundo, el entorno 
Factores personales que inciden en el bajo rendimiento académico de los estudiantes de geometría

familiar, el contexto socioeconómico, las variables demográficas (sexo, edad, estado civil) y la escolaridad de los padres. Este autor concluye que al conocer las autoridades universitarias sobre estos factores podrían diseñar políticas para mejorar la calidad académica de los estudiantes de pre-grado.

Si bien es cierto que para Acuña (2013), un eficiente rendimiento académico representa el resultado de diversas etapas del proceso educativo, en el que participan y se requiere de los esfuerzos de los estudiantes, profesores y comunidad. Incluso facilita el establecimiento de estándares educativos, ya que se pueden medir diversos aspectos determinantes del desempeño estudiantil además del cognoscitivo. Por tal motivo, García (2013) afirma que son varios los factores determinantes del rendimiento académico estudiantil, pues se caracteriza por su carácter multidimensional y, según este autor, gran parte de las investigaciones que han abordado este tema han considerado tres variables: de índole personal, social e institucional.

No obstante, Tejedor y García-Valcárcel (2007)han clasificado en tres categorías las causas del bajo rendimiento estudiantil: institucionales, relacionadas con el profesor y relacionadas con el propio estudiante. Por tal motivo, Lamas (2015), concluye que "para dar respuesta a la preocupación social del bajo rendimiento académico de los estudiantes, se deben analizar distintas variables que puedan influir en dicho rendimiento" (p.343).

De esta manera, Montes y Lerner (2011), consideran que el rendimiento académico de los estudiantes puede ser explicado bajo cinco dimensiones: la académica, la económica, la familiar, la personal y la institucional. Representando los factores o determinantes personales el núcleo de la personalidad del estudiante, protagonista principal del rendimiento académico estudiantil en cualquier nivel educativo, aunque diversos investigadores incluyan en sus estudios distintos indicadores para medirlos. Al respecto, García (2013), manifiesta que los 
Niorka Medina; Johan Fereira y Robinson Marzol

Telos Vol. 20, No. 1 (2018). 4-28.

determinantes personales están constituidos por: competencias cognitivas, motivación, condiciones cognitivas, autoconcepto académico, autoeficacia percibida, bienestar psicológico, asistencia a clases, aptitudes, sexo, formación académica previa a la universidad y la nota de acceso a la universidad.

Ahora bien, los factores personales, denominados por algunos autores dimensión personal, además de asociarlos a los factores individuales o psicológicos del rendimiento académico; en esta investigación incluyen: la motivación, las habilidades sociales y comunicativas así como las cualidades personales.

Autoras como Montes y Lerner (2011) asocian las habilidades comunicativas con: disposición para pedir ayuda, expresar ideas y proponer soluciones para sus preocupaciones, escuchando los puntos de vista de familiares, profesores, compañeros y amigos; destreza para integrarse con compañeros para estudiar. Mientras que las habilidades sociales la relacionan con la influencia tanto positiva o negativa de los amigos, las relaciones de pareja y la responsabilidad con las actividades académicas incluyendo el hecho de afrontar algún duelo personal durante su estadía en la Universidad.

En lo referente a las cualidades personales, se buscó conocer la percepción de los estudiantes acerca de las capacidades para: concentrarse, prestar atención y comprender, controlarse frente a situaciones difíciles y la capacidad para planificar y utilizar el tiempo de forma acorde a las exigencias de sus compromisos académicos. Otro aspecto tomado en cuenta fue la motivación que tienen los estudiantes para continuar sus estudios universitarios, contemplando para ello los valores de superación personal orientados hacia el ejercicio autónomo del logro de aspiraciones propias o de autoafirmación, o del núcleo familiar, o de acuerdo con valores instituidos en el entorno socioeconómico cultural.

\section{Metodología}

El abordaje metodológico de la investigación fue de tipo descriptiva ya que se identifican, describen y analizan los factores personales considerados por los 
Factores personales que inciden en el bajo rendimiento académico de los estudiantes de geometría

estudiantes de la cátedra de Geometría que intervienen en su bajo rendimiento académico. En cuanto al diseño de investigación, fue de campo, debido a que la información se recogió directamente en la facultad de Ingeniería de la Universidad del Zulia; transversal, puesto que los datos son recabados en un solo momento en el segundo periodo del año 2014; para el análisis de los datos obtenidos se siguió una metodología cuantitativa.

Dado que el propósito general de este estudio, fue determinar los factores personales que inciden en el bajo rendimiento de los estudiantes de geometría en la facultad de Ingeniería, se optó por una muestra de 200 estudiantes repitientes inscritos en la cátedra de geometría en el segundo periodo del año 2014 en la facultad de Ingeniería de la Universidad del Zulia. Tal muestra se caracterizó por ser estudiantes pertenecientes a cinco (5) secciones de las ocho (8) secciones de geometría de estudiantes repitientes dictadas durante ese periodo y cursantes de las siete escuelas de la facultad de ingeniería. Esta cátedra está ubicada en el primer semestre de todas las escuelas de ingeniería, de cinco horas por semana, en un semestre de 14 semanas, con 800 alumnos inscritos en la materia para este periodo y una población de 340 estudiantes repitientes.

Para la recolección de datos se utilizó la técnica de la encuesta, utilizando como instrumento un cuestionario denominado CEST dirigido a estudiantes, basado en el cuestionario diseñado por Montes y Lerner (2011) para estudiantes de pregrado de la Universidad de EAFIT conocido como Dimensiones del Rendimiento Académico (DRA), constituido en la sección de Factores Personales por 8 ítems de selección simple y escala de tipo Likert que midieron los siguientes factores personales propuestos por dichas autoras: Datos Personales, Habilidades y Motivación. Los ítems de escala Likert se valoraron de 1 a 5; en todos los casos, indicando la cualidad con la que describe un estudiante las perspectivas sobre sus habilidades comunicativas, la integración con el entorno que le rodea y sus cualidades 
Niorka Medina; Johan Fereira y Robinson Marzol

Telos Vol. 20, No. 1 (2018). 4-28.

personales, así la mayor puntuación mostraba que el estudiante tiene una alta perspectiva sobre sus habilidades. Las alternativas de respuesta fueron: Pésima (1), Mala (2), Regular (3), Buena (4), Excelente (5).

Las relaciones de pareja y los duelos también se indagaron como aspectos personales de posible influencia en el rendimiento académico, es decir se pretendía conocer cómo se ve afectado el rendimiento académico de los estudiantes por asuntos relacionados con su vida afectiva y de relaciones de pareja, lo cual se plasmó en los ítems 6 y 7.

Con respecto a la aplicación del instrumento, a los profesores de la cátedra de Geometría para su inclusión voluntaria en la investigación. El cuestionario se administró en horario docente, el tiempo total medio para responderlo fue de 20 minutos. No se presentaron errores de interpretación en ninguno de los ítems del cuestionario. El proceso estadístico de los datos obtenidos en la recolección se realizó mediante el programa SPSS versión 20, con el cual se hizo un análisis descriptivo con frecuencias relativas porcentuales para cada factor personal.

Además, basándose en la media aritmética de las dimensiones que componen los factores personales se ubicaron los resultados en los siguientes baremos, véase Tabla No.1, dándole respuesta al propósito de la investigación. Se utilizaron dos baremos: las primeras dimensiones son variables discretas cuyos valores varían de acuerdo al número de opciones de respuesta, se creó un baremo en el que se ubicó la media aritmética una vez que se reorganizaron y recodificaron las variables dentro de un rango de 1 a 2 realizando las interpolaciones necesarias para lograr ubicar la media aritmética en una de las categorías de la variable según el baremo (alta, media y baja). Mientras que, para la dimensión Habilidades se empleó una escala Likert por lo que se creó un baremo con rango entre 1 y 5, se recodificó la variable realizando luego las interpolaciones requeridas para poder ubicar a la media aritmética en una de las tres categorías de respuesta para la variable de investigación. 
Factores personales que inciden en el bajo rendimiento académico de los estudiantes de geometría

\section{Tabla No.1}

\section{Baremo de interpretación de respuesta para las dimensiones Habilidad}

\begin{tabular}{|c|c|c|} 
Dimensión a Medir & $\begin{array}{c}\text { Rango del Baremo } \\
\text { para la Media } \\
\text { Aritmética }\end{array}$ & $\begin{array}{c}\text { Categoría para el Factor } \\
\text { Personal }\end{array}$ \\
\hline $\begin{array}{c}\text { Datos Personales, Situaciones } \\
\text { Personales, Relación de Pareja y } \\
\text { Motivación para seguir estudios } \\
\text { universitarios }\end{array}$ & $1,67-2,00$ & Alta Intervención \\
\cline { 2 - 3 } & $1,34-1,66$ & Mediana Intervención \\
\hline Habilidades & $1,00-1,33$ & Baja Intervención \\
\hline & $3,67-5,00$ & Alta Intervención \\
\cline { 2 - 3 } & $2,34-3,66$ & Mediana Intervención \\
\cline { 2 - 3 } & $1,00-2,33$ & Baja Intervención \\
\hline
\end{tabular}

\section{Fuente: Elaboración Propia}

\section{Resultados}

Tomando en cuenta los resultados presentados en la Tabla No.2, se observa que el $43,5 \%$ de los estudiantes han repetido más de dos veces la asignatura. Entre los resultados generales que se destacan en los datos personales de los estudiantes encuestados se encuentra que: la mayoría son del género masculino (53\%), sus edades oscilan entre 20 y 22 años (54\%), se encuentran solteros (94,5\%) y estudian en las escuelas de industrial y eléctrica (21,5 y 21\%, respectivamente), véase la Tabla No. 3. Se ubicaron en el baremo las medias aritméticas, como medida de tendencia central, obtenidas de acuerdo con el análisis estadístico descriptivo para cada una de los indicadores que constituyen a la dimensión Datos Personales, esta tabla resume ese análisis. De acuerdo con el baremo, los datos personales de los estudiantes no influyen significativamente en el bajo rendimiento académico ya que tienen una baja intervención, distinguiéndose quizás los indicadores género y escuela en la que están inscritos con una mediana influencia. 
Niorka Medina; Johan Fereira y Robinson Marzol

Telos Vol. 20, No. 1 (2018). 4-28.

Tabla No. 2

Resultados de la Repitencia en la asignatura Geometría

\begin{tabular}{|c|c|c|c|}
\hline \multicolumn{2}{|c|}{$\begin{array}{l}\text { Repitencia de la asignatura } \\
\text { Geometría }\end{array}$} & Frecuencia & Porcentaje \\
\hline \multirow{4}{*}{ Válidos } & una vez & 66 & 33,0 \\
\hline & dos veces & 47 & 23,5 \\
\hline & $\begin{array}{l}\text { más de dos } \\
\text { veces }\end{array}$ & 87 & 43,5 \\
\hline & Total & 200 & 100,0 \\
\hline
\end{tabular}

Fuente: Elaboración Propia

Tabla No. 3

Resultados de los Datos Personales

\begin{tabular}{|c|c|c|c|c|c|}
\hline \multicolumn{2}{|c|}{ Indicadores/DIMENSIÓN } & Frecuencia & Porcentaje & $\begin{array}{c}\text { Media } \\
\text { Aritmética }\end{array}$ & $\begin{array}{c}\text { Categoría según } \\
\text { Baremo }\end{array}$ \\
\hline Género & $\begin{array}{l}\text { Masculino } \\
\text { Femenino }\end{array}$ & $\begin{array}{r}106 \\
94\end{array}$ & $\begin{array}{l}53,0 \\
47,0\end{array}$ & 1,47 & $\begin{array}{r}\text { Mediana } \\
\text { Intervención }\end{array}$ \\
\hline Edad & $\begin{array}{l}\text { Entre } 17 \text { y } 19 \\
\text { Entre } 20 \text { y } 22 \\
23 \text { y más }\end{array}$ & $\begin{array}{r}80 \\
108 \\
12\end{array}$ & $\begin{array}{r}40,0 \\
54,0 \\
6,0\end{array}$ & 1,33 & Baja Intervención \\
\hline $\begin{array}{l}\text { Estado } \\
\text { Civil }\end{array}$ & $\begin{array}{l}\text { Soltero } \\
\text { Casado }\end{array}$ & $\begin{array}{r}189 \\
11\end{array}$ & $\begin{array}{r}94,5 \\
5,5\end{array}$ & 1,06 & Baja Intervención \\
\hline Escuela & $\begin{array}{l}\text { Petróleo } \\
\text { Química } \\
\text { Geodesia } \\
\text { Civil } \\
\text { Industrial } \\
\text { Mecánica } \\
\text { Eléctrica }\end{array}$ & $\begin{array}{l}35 \\
11 \\
28 \\
18 \\
43 \\
23 \\
42\end{array}$ & $\begin{array}{r}17,5 \\
5,5 \\
14,0 \\
9,0 \\
21,5 \\
11,5 \\
21,0\end{array}$ & 1,43 & $\begin{array}{r}\text { Mediana } \\
\text { Intervención }\end{array}$ \\
\hline \multicolumn{4}{|c|}{ DATOS PERSONALES } & 1,32 & Baja Intervención \\
\hline
\end{tabular}

Fuente: Elaboración Propia 
Factores personales que inciden en el bajo rendimiento académico de los estudiantes de geometría

Las habilidades comunicativas, sociales y personales forman parte de la vida de todo individuo y se presume que actúen positivamente en su desempeño académico. Sin embargo, ante un bajo rendimiento de los estudiantes, los resultados se muestran en la Tabla No. 4, los cuales responden a las percepciones sobre sus habilidades comunicativas, la integración con el entorno que le rodea y las cualidades personales. Según tales resultados, los estudiantes calificaron como su pésima habilidad comunicativa(56\%) "el diálogo con amigos y profesores sobre situaciones personales", la segunda peor habilidad $(24,5 \%)$ fue "la capacidad de aportar propuestas, soluciones, alternativas e inconformidades"; en contraste con solo el $33,5 \%$ de los estudiantes que se les hace fácil "pedir ayuda cuando se tiene dificultad".

Entre las habilidades sociales que destacan por considerarlas las pésimas habilidades desarrolladas por los estudiantes de bajo rendimiento están "la facilidad para darse a conocer a otras personas" (15\%), seguida por "la capacidad para organizar actividades" (12,5\%), ante una excelente "influencia positiva de amigos o conocidos en su vida" (31\%). La peor calificación para las cualidades personales fue "su capacidad de planificar y utilizar el tiempo de manera efectiva" (70\%), seguida de "el control personal frente a situaciones difíciles" (30\%); destacándose como su mejor cualidad personal "su responsabilidad con las actividades" (33\%). 


\begin{tabular}{|c|c|c|c|c|c|c|c|c|c|c|c|}
\hline \multicolumn{12}{|c|}{$\begin{array}{c}\text { Tabla No.4 } \\
\text { Resultados de las Habilidades }\end{array}$} \\
\hline \multirow{2}{*}{\multicolumn{2}{|c|}{ Habilidades }} & \multicolumn{2}{|c|}{ Pésima } & \multicolumn{2}{|c|}{ Mala } & \multicolumn{2}{|c|}{ Regular } & \multicolumn{2}{|c|}{ Buena } & \multicolumn{2}{|c|}{ Excelente } \\
\hline & & $\mathrm{fr}$ & $\%$ & fr & $\%$ & fr & $\%$ & fr & $\%$ & $\mathrm{fr}$ & $\%$ \\
\hline \multirow{5}{*}{ 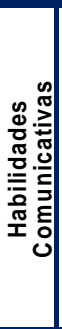 } & $\begin{array}{l}\text { La facilidad para pedir ayuda cuando se } \\
\text { tiene dificultad }\end{array}$ & 8 & 4,0 & 31 & 15,5 & 44 & 22,0 & 44 & 22,0 & 67 & 33,5 \\
\hline & $\begin{array}{l}\text { La capacidad para explicar sus ideas } \\
\text { claramente }\end{array}$ & 8 & 4,0 & 35 & 17,5 & 56 & 28,0 & 46 & 23,0 & 23 & 11,5 \\
\hline & $\begin{array}{l}\text { La capacidad para integrarse con otros } \\
\text { compañeros para estudiar }\end{array}$ & 23 & 11,5 & 31 & 15,5 & 34 & 17,0 & 59 & 29,5 & 53 & 26,5 \\
\hline & $\begin{array}{l}\text { El diálogo con amigos y profesores sobre } \\
\text { situaciones personales }\end{array}$ & 112 & 56,0 & 63 & 31,5 & 24 & 12,0 & 15 & 7,5 & 20 & 10,0 \\
\hline & $\begin{array}{l}\text { La capacidad de aportar propuestas, } \\
\text { soluciones, alternativas e inconformidades }\end{array}$ & 49 & 24,5 & 40 & 20,0 & 42 & 21,0 & 36 & 18,0 & 37 & 18,5 \\
\hline \multirow{6}{*}{ 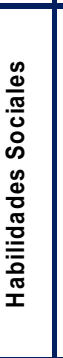 } & $\begin{array}{l}\text { La influencia positiva de amigos } 0 \\
\text { conocidos en su vida }\end{array}$ & 13 & 6,5 & 19 & 9,5 & 16 & 8,0 & 51 & 25,5 & 62 & 31,0 \\
\hline & $\begin{array}{l}\text { La influencia negativa de amigos } 0 \\
\text { conocidos en su vida }\end{array}$ & 119 & 59,5 & 75 & 37,5 & 21 & 10,5 & 0 & 0,0 & 3 & 1,5 \\
\hline & $\begin{array}{l}\text { La consideración de sus compañeros } \\
\text { como amigos }\end{array}$ & 13 & 6,5 & 29 & 14,5 & 40 & 20,0 & 32 & 16,0 & 45 & 22,5 \\
\hline & $\begin{array}{l}\text { Su disposición para considerar puntos de } \\
\text { vista de los demás }\end{array}$ & 0 & 0,0 & 19 & 9,5 & 43 & 21,5 & 46 & 23,0 & 37 & 18,5 \\
\hline & Su capacidad para organizar actividades & 25 & 12,5 & 37 & 18,5 & 36 & 18,0 & 33 & 16,5 & 31 & 15,5 \\
\hline & $\begin{array}{l}\text { La facilidad para darse a conocer a otras } \\
\text { personas }\end{array}$ & 30 & 15,0 & 21 & 10,5 & 44 & 22,0 & 38 & 19,0 & 22 & 11,0 \\
\hline \multirow{4}{*}{ 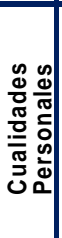 } & $\begin{array}{l}\text { La facilidad para prestar atención y } \\
\text { comprender lo que dicen }\end{array}$ & 0 & 0,0 & 29 & 14,5 & 46 & 23,0 & 60 & 30,0 & 56 & 28,0 \\
\hline & $\begin{array}{l}\text { El control personal frente a situaciones } \\
\text { difíciles }\end{array}$ & 60 & 30,0 & 48 & 24,0 & 44 & 22,0 & 50 & 25,0 & 61 & 30,5 \\
\hline & Su responsabilidad con las actividades & 0 & 0,0 & 37 & 18,5 & 41 & 20,5 & 55 & 27,5 & 66 & 33,0 \\
\hline & $\begin{array}{l}\text { Su capacidad de planificar y utilizar el } \\
\text { tiempo de manera efectiva }\end{array}$ & 140 & 70,0 & 86 & 43,0 & 69 & 34,5 & 35 & 17,5 & 17 & 8,5 \\
\hline
\end{tabular}

Fuente: Elaboración Propia

En la siguiente tabla se ubican los resultados de la media aritmética de la dimensión Habilidades y cada uno de sus indicadores en el baremo de la investigación, en la que se observa que la dimensión Habilidades presenta una intervención mediana en el bajo rendimiento de los estudiantes. 
Factores personales que inciden en el bajo rendimiento académico de los estudiantes de geometría

Tabla No. 5

Habilidades de los Estudiantes

\begin{tabular}{|l|r|l|}
\hline \multicolumn{1}{|c}{ Indicadores/DIMENSIÓN } & \multicolumn{1}{c|}{$\begin{array}{c}\text { Media } \\
\text { Aritmética }\end{array}$} & $\begin{array}{c}\text { Categoría según } \\
\text { Baremo }\end{array}$ \\
\hline Habilidades Comunicativas & 3,02 & Mediana Intervención \\
\hline Habilidades Sociales & 3,20 & Mediana Intervención \\
Cualidades Personales & 3,60 & Mediana Intervención \\
\hline HABILIDADES & 3,27 & Mediana Intervención \\
\hline
\end{tabular}

Fuente: Elaboración Propia

Asimismo, se investigó sobre vivencias o situaciones personales significativas como las relaciones de pareja y el duelo lo que permitiría analizar aspectos personales de posible influencia en el bajo rendimiento académico del estudiante. De esta manera resultó que en los duelos, la mayoría de los estudiantes (37\%) no ha tenido que afrontar situaciones de duelo durante sus estudios universitarios, el más significativo se refiere a duelos por quiebra económica de la familia (25\%), seguido por la muerte de alguna persona significativa $(21,5 \%)$ y luego por ruptura amorosa $(16,5 \%)$, el $70 \%$ de los estudiantes no han establecido una relación de pareja durante su permanencia en la universidad (ver Tabla No. 6 y 7). En estas tablas se distingue que ambas dimensiones tienen una alta intervención en el bajo rendimiento académico.

\section{Tabla No.6}

Resultados de Vivencias Personales Significativas

\begin{tabular}{|c|c|c|c|c|}
\hline Indicadores/DIMENSIÓN & Frecuencia & Porcentaje & $\begin{array}{c}\text { Media } \\
\text { Aritmética }\end{array}$ & $\begin{array}{c}\text { Categoría según } \\
\text { Baremo }\end{array}$ \\
\hline Ruptura Amorosa & 33 & 16,5 & 1,79 & Alta Intervención \\
\hline Muerte de alguna persona significativa & 43 & 21,5 & 1,73 & Alta Intervención \\
\hline Quiebra económica de la familia & 50 & 25,0 & 1,69 & Alta Intervención \\
\hline Ninguna & 74 & 37,0 & 1,54 & $\begin{array}{r}\text { Mediana } \\
\text { Intervención }\end{array}$ \\
\hline \multicolumn{3}{|l|}{ SITUACIONES PERSONALES } & 1,69 & Alta Intervención \\
\hline
\end{tabular}

Fuente: Elaboración Propia 


\section{Tabla No. 7}

Resultados de Relación en Pareja

\begin{tabular}{|c|c|c|c|c|}
\hline $\begin{array}{l}\text { RELACIÓN EN } \\
\text { PAREJA }\end{array}$ & Frecuencia & Porcentaje & $\begin{array}{c}\text { Media } \\
\text { Aritmética }\end{array}$ & $\begin{array}{c}\text { Categoría según } \\
\text { Baremo }\end{array}$ \\
\hline & 60 & 30,0 & \multirow{2}{*}{1,70} & \multirow{2}{*}{ Alta Intervención } \\
\hline no & 140 & 70,0 & & \\
\hline
\end{tabular}

Fuente: Elaboración Propia

La Tabla No.8 muestra, que la mayor motivación para seguir estudio universitarios es "por superación personal/logro personal"(14,93\%), seguida "porque quiere tener una vida más cómoda en el futuro" $(12,44 \%)$ y "porque graduarse es una oportunidad para desempeñarse como profesional en el exterior" $(11,94 \%)$ como los tres primeros lugares, respectivamente. Teniendo en cuenta como los dos menos importantes: "porque tengo beca o subsidios económicos" y "a perder el tiempo" con un $0,5 \%$ para cada uno. Se puede resaltar que la motivación para seguir estudios universitarios tiene una mediana intervención en el bajo rendimiento según la ubicación en el baremo de la media aritmética. 
Factores personales que inciden en el bajo rendimiento académico de los estudiantes de geometría

Tabla No. 8

Resultados de Motivación para seguir estudios universitarios

\begin{tabular}{|c|c|c|c|c|}
\hline Indicadores/DIMENSIÓN & Frecuencia & Porcentaje & $\begin{array}{c}\text { Media } \\
\text { Aritmética }\end{array}$ & $\begin{array}{l}\text { Categoría } \\
\text { según Baremo }\end{array}$ \\
\hline Porque mis padres/ familia dicen que es muy & 11 & 5,47 & 1,69 & Alta \\
\hline importante & & & & Intervención \\
\hline Porque me encuentro con mis amigos & 5 & 2,47 & 1,85 & $\begin{array}{c}\text { Alta } \\
\text { Intervención }\end{array}$ \\
\hline Porque graduarse es una obligación social & 5 & 2,49 & 1,84 & Alta \\
\hline Porque tener el título da prestigio & 10 & 4,98 & 1,71 & $\begin{array}{l}\text { Intervencion } \\
\text { Alta }\end{array}$ \\
\hline & & & & Intervención \\
\hline $\begin{array}{l}\text { Porque el ambiente universitario es } \\
\text { aqradable }\end{array}$ & 7 & 3,48 & 1,78 & $\begin{array}{c}\text { Alta } \\
\text { Intervención }\end{array}$ \\
\hline Por superación personal//ogro personal & 30 & 14,93 & 1,11 & $\begin{array}{l}\text { Baja } \\
\text { Intervención }\end{array}$ \\
\hline $\begin{array}{l}\text { Porque quiero demostrarme a mí mismo que } \\
\text { soy una persona inteligente }\end{array}$ & 19 & 9,45 & 1,44 & $\begin{array}{l}\text { Mediana } \\
\text { Intervención }\end{array}$ \\
\hline $\begin{array}{l}\text { Porque encuentro satisfacción y me gusta } \\
\text { aprender cosas nuevas }\end{array}$ & 22 & 10,95 & 1,34 & $\begin{array}{l}\text { Mediana } \\
\text { Intervención }\end{array}$ \\
\hline $\begin{array}{l}\text { Porque me permitirá escoger un trabajo en la } \\
\text { rama que me gusta }\end{array}$ & 23 & 11,44 & 1,31 & $\begin{array}{l}\text { Baja } \\
\text { Intervención }\end{array}$ \\
\hline $\begin{array}{l}\text { Porque tengo la capacidad económica } \\
\text { (familia con capacidad, mi propia capacidad) }\end{array}$ & 4 & 1,99 & 1,88 & $\begin{array}{c}\text { Alta } \\
\text { Intervención }\end{array}$ \\
\hline Porque tengo beca o subsidios económicos & 1 & 0,50 & 1,98 & $\begin{array}{c}\text { Alta } \\
\text { Intervención }\end{array}$ \\
\hline $\begin{array}{l}\text { Porque quiero tener una vida más cómoda } \\
\text { en el futuro }\end{array}$ & 25 & 12,44 & 1,25 & $\begin{array}{l}\text { Baja } \\
\text { Intervención }\end{array}$ \\
\hline A perder el tiempo & 1 & 0,50 & 1,98 & $\begin{array}{l}\text { Alta } \\
\text { Intervención }\end{array}$ \\
\hline $\begin{array}{l}\text { Porque graduarse es una oportunidad para } \\
\text { desempeñarse como profesional en el } \\
\text { exterior }\end{array}$ & 24 & 11,94 & 1,29 & $\begin{array}{l}\text { Baja } \\
\text { Intervención }\end{array}$ \\
\hline Para trabajar fuera del país & 14 & 6,97 & 1,58 & $\begin{array}{l}\text { Mediana } \\
\text { Intervención }\end{array}$ \\
\hline \multicolumn{3}{|c|}{ MOTIVACIÓN PARA SEGUIR ESTUDIOS UNIVERSITARIOS } & 1,60 & $\begin{array}{c}\text { Mediana } \\
\text { Intervención }\end{array}$ \\
\hline
\end{tabular}

Fuente: Elaboración Propia

Finalmente, se presenta en la Tabla No. 9 el resumen de los resultados de cada uno de sus dimensiones y su intervención en bajo rendimiento de los estudiantes, en el 
que predominan las situaciones personales y relaciones en pareja en el bajo rendimiento, siendo los datos personales el factor con menos influencia en tal rendimiento.

\section{Tabla No. 9 \\ Resultados de los Factores Personales}

\begin{tabular}{|l|r|r|}
\hline \multicolumn{1}{|c|}{ DIMENSIÓNES } & $\begin{array}{c}\text { Media } \\
\text { Aritmética }\end{array}$ & \multicolumn{1}{c|}{$\begin{array}{c}\text { Categoría según } \\
\text { Baremo }\end{array}$} \\
\hline Datos Personales & 1,32 & Baja Intervención \\
Habilidades & 3,27 & Mediana Intervención \\
Situaciones Personales Significativas & 1,69 & Alta Intervención \\
Relaciones de Pareja & 1,70 & Alta Intervención \\
Motivación para seguir estudios universitarios & 1,60 & Mediana Intervención \\
\hline
\end{tabular}

Fuente: Elaboración Propia

\section{Conclusiones}

Las causas relacionadas para explicar el bajo rendimiento académico son diversas y plurales, incluso asociadas con la edad en que ingresan los estudiantes a la universidad, la selección de su carrera, sus habilidades y motivación. No obstante, los jóvenes estudiantes en pocas oportunidades están conscientes de su responsabilidad en su posible "fracaso académico" y suelen responsabilizar a la institución ya sus profesores, especialmente en cátedras como Geometría, área de la matemática que exige para su comprensión: estudio, orden, rigurosidad, y un trabajo dedicado y continúo.

En el momento en que el estudiante no admite su compromiso académico y no adopta una actitud crítica frente a sus deberes universitarios se genera un fracaso reiterativo y puede crearse un rechazo hacia el objeto de conocimiento incluso hacia su entorno universitario. En consecuencia, las instituciones universitarias se ven en la necesidad urgente de reflexionar sobre su responsabilidad en esta realidad e implementar estrategias para mejorar y buscar soluciones factibles conformes a la calidad de la educación. 
Factores personales que inciden en el bajo rendimiento académico de los estudiantes de geometría

Tomando en consideración lo que la literatura manifiesta sobre el análisis del bajo rendimiento académico de los estudiantes, la cual involucra las capacidades, aptitudes y la motivación con que cuenta el individuo para la búsqueda del saber, parte de estas características estuvieron contenidas en los factores personales, los cuales se relacionaron con las habilidades tanto sociales como comunicativas e incluso cualidades personales que comprenden comportamientos verbales y no verbales.

Aspectos como la ansiedad, que producen las evaluaciones antes y durante la misma, puede ocasionar bloqueos al estudiante, confusión y temor en sus decisiones al momento de responder cualquier pregunta durante la prueba, asuntos relacionados con su vida afectiva y de relaciones de pareja, la manera en cómo puede afectar haber sufrido algún duelo, una ruptura amorosa durante su proceso formativo también resultaron relevantes. Finalmente, la motivación; en la que se hace referencia a la promesa de lucro al culminar su carrera, el reconocimiento social y familiar, el cumplimiento de objetivos personales o familiares, entre otros.

Con respecto a los resultados de esta investigación, los mismos señalan que los factores personales medianamente intervienen en el bajo rendimiento de los estudiantes de geometría, siendo los datos personales los de menor intervención, los cuales incluyen: género, edad, estado civil, escuela que cursa. Mientras que, las habilidades constituidas por: las comunicativas, sociales y cualidades personales, cuentan con una mediana intervención aunque los estudiantes se perciben positivamente en cuanto a sus habilidades sociales, su vida afectiva y sus relaciones con compañeros, siendo reservados al exponer sus asuntos personales y en considerar a sus compañeros como amigos.

Además de ello, resultan ser pésimos a la hora de planificar y utilizar el tiempo de manera efectiva. Por lo tanto, se genera una limitante en su desempeño académico, pues con la óptima utilización de los recursos educativos, y el tiempo es parte de ello, se lograría una mayor eficacia en el proceso de aprendizaje. 
Niorka Medina; Johan Fereira y Robinson Marzol

Telos Vol. 20, No. 1 (2018). 4-28.

La motivación para seguir estudios universitarios resultó tener una intervención mediana; sin embargo hay que destacar que las razones que tienen alta intervención en el bajo rendimiento son "a perder el tiempo" y "porque tengo beca o subsidios económicos", lo que lleva a interpretar que estos estudiantes necesitan mejores incentivos y tener un mayor compromiso individual para llevar sus estudios con éxito. Por último, las vivencias o situaciones personales significativas que incluyen duelos, rupturas amorosas y quiebra económica aunada al establecimiento de una relación en pareja durante su permanencia en la universidad, representan los factores con mayor intervención en el bajo rendimiento académico.

En líneas generales, el rendimiento académico es el resultado del complicado universo que rodea al estudiante, y representa un indicador de la eficiencia de un sistema educativo en cualquiera de sus niveles, pues suministra la información necesaria para activar cualquier proceso evaluativo requerido para lograr una educación de calidad. Desde un punto de vista práctico, se suele asociar rendimiento académico con calificaciones o notas, para lo cual están presente múltiples determinantes o factores que influyen en el mismo, los cuales pueden ser inherentes al alumno, al profesor y a la institución educativa.

De acuerdo con el manejo de las calificaciones y en la mayoría de las instituciones universitarias se ha señalado que el rendimiento académico se puede clasificar de bajo, medio y alto. Existen estudiantes de bajo rendimiento que trabajan por debajo de su capacidad en todas las materias que cursan y otros que solo lo hacen en algunas. Este último es conocido como bajo rendimiento especifico y se puede generar por problemas específicos en el aprendizaje o bien como consecuencia de alguna experiencia traumática.

En este sentido, se concluye, que con esta investigación se obtendrá información valiosa para la planificación e implementación de estrategias de apoyo académico y propuestas para mejorar el aprendizaje facilitando la retroalimentación en los procesos de enseñanza-aprendizaje; optimizando el rendimiento académico de 
Factores personales que inciden en el bajo rendimiento académico de los estudiantes de geometría

los estudiantes de geometría. Aun cuando, existen una serie de factores determinantes a considerar para el análisis del bajo rendimiento académico, el presente artículo representa un buen acercamiento para explicar una parte de este análisis, de allí la necesidad de profundizar en el perfil del estudiante de geometría para luego poder determinar los factores que afectan directamente a su rendimiento y crear programas de intervención para mejorarlo.

Por tal razón, se sugiere al Departamento de Matemática de la Facultad de Ingeniería de la Universidad del Zulia llevar a cabo investigaciones sobre las causas del bajo rendimiento académico de estos estudiantes abordando todos los factores involucrados e implementar las soluciones convenientes para solventar en el menor tiempo posible estas dificultades realizando un seguimiento a los mismos; obviamente con la colaboración de los docentes, las autoridades y principalmente del estudiante, como centro del proceso educativo. Por otro lado, este estudio no intenta ser concluyente sólo pretende apoyar el análisis del bajo rendimiento académico universitario en Venezuela.

\section{Referencias Bibliográficas}

Acuña Espinoza, José. (2013). Autoestima Y Rendimiento Académico de los Estudiantes del X Ciclo 2012 - II de la Escuela Académica Profesional de Educación Primaria y Problemas de Aprendizaje de La Universidad Nacional José Faustino Sánchez Carrión - Huacho. Tesis Para optar el grado académico de Magíster en ciencias de la gestión educativa con mención en pedagogía. Universidad Nacional José Faustino Sánchez Carrión. Perú.

Báez Aumala, Mario. (2013).Factores que determinan el desempeño académico en

Zamorano. Proyecto especial para optar al título de Ingeniero en Agro negocios en el Grado Académico de Licenciatura. Escuela Agrícola Panamericana. Honduras.

Barahona, Planck. (2014). Factores determinantes del rendimiento académico de los estudiantes de la Universidad de Atacama. Revista Estudios Pedagógicos. Volumen XL, No. 1. Chile (Pp. 25-39). 
Boyer, Yennyy Pérez Dalgys. (2011). Necesidades educativas especiales de los alumnos con bajo rendimiento estudiantil en el Instituto Universitario de Tecnología del Oeste "Mariscal Sucre". Revista EDU-FISICA, Volumen 3, No. 7. Cuba (Pp. 1-12).

Congreso de la República de Venezuela. (1999). Ley Orgánica de Educación con su Reglamento. Gaceta Oficial 36.787. Venezuela.

Consejo Universitario de la Universidad del Zulia. (2000). Reglamento de Evaluación

y Rendimiento Estudiantil de la Universidad del Zulia. Venezuela

Garbanzo Vargas, Guiselle. (2007). Factores asociados al rendimiento académico en

estudiantes universitarios, una reflexión desde la calidad de la educación superior pública. Revista Educación, Volumen 31, No.1.Costa Rica (Pp. 43$63)$.

García, Deysi. (2013). Estrategia Didáctica para mejorar el Rendimiento Académico de los estudiantes en la Asignatura Cálculo I de la Facultad Experimental de Ciencias y Tecnología de la Universidad de Carabobo. Trabajo de Grado para optar al título de Magíster en Desarrollo Curricular. Universidad de Carabobo. Venezuela.

García-Cruz, Rubén,Guzmán Saldaña, Rebeca y Martínez Martínez, Juan. (2007). Tres aristas de un triángulo: Bajo Rendimiento Académico, Fracaso Y Deserción Escolar, y un centro: "El Adolescente". Revista Electrónica de Psicología, Volumen2, No. 1. México

Guzmán Brito, Martha. (2012). Modelos predictivos y explicativos del rendimiento académico universitario: caso de una institución privada en México. Tesis Doctoral. Universidad Complutense de Madrid. España.

Hernández, Ana. (2005). El rendimiento académico de las matemáticas en alumnos universitarios. Revista Encuentro Educacional, Volumen 12, No.1, Venezuela (Pp.9-30).

Lamas Héctor. (2015). Sobre el rendimiento escolar. Propósitos y Representaciones, Volumen 3, No. 1, Perú (Pp.313-386).

Martí Ballester, Carmen. (2012). Análisis de los Factores que influyen en el Desempeño Académico de los Alumnos de Contabilidad Financiera a través de Modelos de Elección Binaria. Revista Brasileira de Gestáo de Negócios, Volumen 14, No.45, Brasil (Pp.379-399).

Martínez León, Laura y Paladinez Velez, Merci (2016). Trastornos afectivos y su incidencia en el rendimiento académico de los estudiantes de la escuela de educación básica "Paul Percy Harry Bryan" de la ciudad de Machala perteneciente a la ciudadela el bosque. Tesis de Grado para el título de Licenciado en Ciencias de la Educación, Mención: Psicología Educativa y Orientación Vocacional. Universidad Técnica De Machala. Ecuador.

Miranda, José; Montaño, Borja y Sáez, Miguel. (2012). Causas del bajo rendimiento de los alumnos en la asignatura Historia Económica Mundial y de España. X 
Factores personales que inciden en el bajo rendimiento académico de los estudiantes de geometría

Jornadas Redes de Investigación en Docencia Universitaria. Madrid, España.

Montes Gutiérrez, Isabel y Lerner Matiz, Jeannette. (2011). Rendimiento académico de los estudiantes de pregrado de la Universidad EAFIT. Perspectiva cuantitativa. Extraído de http://www.eafit.edu.co/institucional/calidadeafit/investigacion/Documents/Rendimiento\%20Ac\%C3\%A1demicoPerrspectiva\%20cuantitativa.pdf Consulta 18/01/15

Pitalúa González, Eliodoro. (2012). Estrategias de aprendizaje utilizadas por los estudiantes de Geometría y su relación con el rendimiento académico. Revista Electrónica de Humanidades, Educación y Comunicación Social, REDHECS, Volumen 12, No.7,Venezuela (Pp.114-124).

Rodríguez, Sebastián; Fita, Eva y Torrado, Mercedes. (2004). El rendimiento académico en la transición secundaria-universidad. Revista Educación, No. 334, España (Pp. 391-14).

Secretaria del Consejo de la Facultad de Ingeniería. (2001). Sistema de Evaluación de los Aprendizajes de la Facultad de Ingeniería. Universidad del Zulia. Venezuela.

Tapia Pavón, Adelfo y Tapia Fernández, Santiago. (2012). Predisposiciones que Influyen en el Rendimiento Académico.

Tejedor Tejedor, Francisco y García-Valcárcel Muñoz-Repiso, Ana. (2007). Causas del bajo rendimiento del estudiante universitario (en opinión de los profesores y alumnos). Propuestas de mejora en el marco del EEES. Revista de Educación, No. 342, España (Pp. 443-473). 